\title{
Design of Shock Wave Storage and Test System with Variable Parameters Based on the Sensor of Piezoelectric Circuit
}

\author{
Zhi Li (ii) \\ College of Mathematics and Information Engineering, Chongqing University of Education, Nan'an, 400065 Chongqing, China \\ Correspondence should be addressed to Zhi Li; lizhi@cque.edu.cn
}

Received 8 February 2021; Revised 17 April 2021; Accepted 20 May 2021; Published 15 June 2021

Academic Editor: Wenqing Wu

Copyright @ 2021 Zhi Li. This is an open access article distributed under the Creative Commons Attribution License, which permits unrestricted use, distribution, and reproduction in any medium, provided the original work is properly cited.

\begin{abstract}
With the wide application of science and technology in the field of weapons, shock wave is an important breakthrough point in weapon research, and the storage and testing system of shock wave is a breakthrough point that people pay most attention to at present. Shock wave data storage has the characteristics of large scale, complex structure, low cost efficiency, and strong timeliness. This paper mainly studies the design of shock wave storage test system with variable parameters based on numerical piezoelectric circuit sensor. Based on fluid dynamics simulation theory and numerical simulation method, the normal and concave-convex three-dimensional models of two pressure measuring devices are constructed by using the flow waveform of calculator, and then, the network is divided. The results show that, under the same inlet pressure, the larger the bulge or depression value, the greater the influence on the experimental results. The influence of disk is $10 \%$ higher than that of pen, and the change rate of relative difference is increased by $1.5 \%$ with the increase of concave-convex value. Finally, experiments are carried out in different environments to verify the reliability, survivability, and flexibility. The shock wave storage test system is optimized when the parameters of the digital voltage circuit sensor are variable.
\end{abstract}

\section{Introduction}

The research on the overpressure and impulse of shock wave can provide an important reference for the comparison of the impact damage strength of weapon equipment. At present, there are two kinds of shock wave sensors at home and abroad: piezoresistive sensor and piezoelectric sensor. The biggest disadvantage of piezoresistive sensor is that its silicon element is very sensitive to the light produced by explosion. Even if the light film is attached to the back of the polished silicon chip to reduce light transmission, the strong light generated by the explosion can cause system failure and reduce the reliability of the system. The shortcomings of piezoelectric sensors are as follows: high output obstacles lead to increase of rise time; low tuning frequency makes it impossible to achieve a good negative pressure curve; signal conversion and processing are more complex, requiring high insulation of cables and joints; electromagnetic waves generated in explosion environment are connected; and debris and vibration of mounting bracket can easily cause signal deviation and affect test results. With the development of IC, a new sensor has been developed. The traditional position charge amplifier is placed on it, the signal is not easy to be interfered, and all high interference circuits are sealed, so that the signal can be transmitted for a long distance without affecting the signal quality, which is convenient to use. It is very suitable to use in the test of shock wave overpressure storage test.

With the continuous development of science and technology, testing plays an increasingly important role in scientific experiments, technical research, and mechanical testing. The more accurate the test is, the more feedback and guidance scientific research can be provided. At present, with the rapid development of sensor technology, sensor design, and dynamic calibration principle, the accuracy of sensor measurement results is improving. In a word, an accurate and reliable sensor is essential behind the formation of an automatic detection and control system. Modern electronic technology and computer provide perfect means for transforming and processing information. Great changes are taking place in the field of modern detection and control system. Various sensors are used to detect data and provide 
information. Therefore, the sensor plays an important role. In the current shock wave overpressure test, pen sensor and disk sensor structures have been widely used, but the shape of the pressure sensor which should be placed in the explosion field is not important. In the test, because the fluidity of the forward shock wave is affected by the geometry of the sensor, the measurement accuracy of the peak value of the shock wave overpressure will inevitably be affected. Therefore, it is necessary to test the overpressure data of the shock wave under the extreme pressure environment. Under these circumstances, the urgent problems to be solved in the shock wave overpressure test include the selection of the sensor settings and the installation of the sensor and error correction of test results. Therefore, it is necessary to simulate and test the shape and structure of various commonly used shock wave testing devices to improve the accuracy of test results.

Robert et al. designed a set of integrated sensor storage test system, regulation circuit, and data acquisition circuit design. Based on the principle of storage test technology and the new ICP piezoelectric sensor, the explosion wave overpressure acquisition and storage test system are designed. The piezoelectric module is integrated with the built-in charge amplifier to output the amplified signal. Through the matching operation of OPAMP and max4638, the function of adjustable gain is realized. The corresponding analog voltage is filtered by a LC $\Pi$ filter, and the amplified signal is filtered by a Sallen key second-order filter. Using FPGA to design digital logic control improves the stability and reliability of the test system and reduces the system volume. Under the same test conditions, the duration of shock wave overpressure is directly proportional to the test radius and inversely proportional to the shock wave size and shock wave. However, because they did not have a control group for comparison, the results obtained were not very convincing [1]. Couldrick et al. designed a system including a data storage medium and a shock wave generator. The data storage medium includes cell and multilayer. Each unit is configured to store information in it. In the same layer of a plurality of layers of a data storage medium, at least two units are arranged in a horizontal plane, and in different layers of a plurality of layers of a data storage medium, at least two units are arranged in a vertical plane. The shock wave generator is configured to generate a shock wave signal propagating through one of the multilayer layers of the data storage medium. When the shock wave signal passes through the target cell, the target cell in the layer stores information in response to the light beam emitted from the transmitter targeting the target unit. After the shock wave signal exits through the target unit, the target unit will retain the information. However, because the shock signal is not stable, the cell information stored in the experiment is not very accurate [2]. Tobin and Hargather propose a wireless passive pressure sensor based on a miniature flexible pressure-sensitive capacitor, which is specially designed to monitor the intracranial pressure (ICP) of patients with craniocerebral trauma. A flexible varistor capacitor is characterized by creatively utilizing PDMS wrinkles generated spontaneously in the process of PDMS etching to construct cavity structure to improve sensi- tivity. In addition, the flexible reader coil for signal reading has been optimized to better couple the sensor in a sufficiently large detection range for ICP monitoring. In addition, because of its flexibility and thin thickness, flexible varistor can adapt to the bending morphology of the skull and dura. Compared with the previous reports, the sensor has lower resonance frequency, higher quality factor, and better sensitivity, but it is also more complex, and it is difficult to control when using [3].

The innovation of this paper lies in the three-dimensional modeling and simulation of the external flow field of the pen type and disc type shock wave overpressure testing device, further carries out simulation and comparative analysis on the influence of the concave-convex and angle installation modes on the flow field, and summarizes the comparison results $[4,5]$.

\section{Shock Wave Storage Test Method Based on Variable Parameters}

2.1. Formation Method of Explosion Shock Wave. The initial state of many substances contains huge energy. In some cases, the state of materials changes suddenly, and the rapid release process of internal energy is called explosion. Generally speaking, explosion refers to a kind of chemical reaction, in which one substance is converted into high-temperature and high-pressure gas, and the reaction time is very short, accompanied by high temperature of about $4000^{\circ} \mathrm{C}$ and tens of thousands of atmospheric pressure, shock wave. The thickness of the wave front is very thin, and the amplitude of the time field is less than 2 ns. If the strong shock wave lacks strong airflow, it will rapidly decompose into weak shock wave. When the explosion product moves a certain distance, the pressure will drop to atmospheric pressure when the explosion does not occur, and the explosion product is still far away [6]. The pressure is less than that of the atmosphere, resulting in negative pressure area. When the energy of the explosion is exhausted, the negative pressure time is over. The pressure rises slowly; the surrounding material shrinks and reaches equilibrium after several cycles. The damage effect of explosion shock wave depends on the pressure in front of the shock wave, that is, the maximum overpressure dynamic value of shock wave $\Delta U$ :

$$
\begin{gathered}
\Delta U=0.085 \frac{\sqrt[3]{E}}{T}+0.28\left(\frac{\sqrt[3]{E}}{T}\right)^{2}+0.8\left(\frac{\sqrt[3]{E}}{T}\right)^{3} \\
y_{i t}=\alpha_{0}+D \max _{i t}+\alpha_{2} X_{i t}+\mu_{i}+\eta_{t}+\alpha_{i t}
\end{gathered}
$$

In the formula, $E$ is the charge weight and $t$ is the detonation distance [7].

\subsection{Method for Estimating Shock Wave Parameters}

2.2.1. Calculation Method of Maximum Overpressure Value of Shock Wave Produced by Air Explosion. At present, there 
is no direct method to measure the specific impulse when evaluating the effect of shock wave. Generally, the method of numerical integration is used to measure the specific impulse based on the pressure signal curve. The specific impulse of explosion shock wave refers to the real statistical data of pressure and time when explosion occurs in the experimental pressure model $[8,9]$. When air explosion occurs, the main factors related to overpressure peak value $\Delta U$ include explosion energy $R_{0}$, initial pressure $P_{0}$, initial air density $\rho_{0}$, and combustion distance $t$ [10]. Therefore, the maximum value can be expressed as

$$
\Delta A=G\left(R_{0}, P_{0}, \rho_{0}, t\right) .
$$

Through theoretical analysis and numerical calculation, the maximum value of shock wave overpressure can be expressed as a function in the form of charge weight and ignition distance of $\sqrt[3]{\widetilde{E}} / t[11,12]$ :

$$
\Delta A=G\left(\frac{\sqrt[3]{\tilde{E}}}{t}\right) .
$$

The above function expansion is transformed into a power function form:

$$
\begin{gathered}
\Delta A=G\left(\frac{\sqrt[3]{\widetilde{E}}}{t}\right)=S_{O}+\frac{S_{1}}{t}+\frac{S_{2}}{t^{2}}+\cdots, \\
C(k)=\left[\zeta_{1} c_{1}(t)+\zeta_{2} c_{2}(k)+\zeta_{3} c_{3}(k)\right. \\
\left.+\zeta_{4} c_{4}(k)+\zeta_{5} c_{5}(k)+\zeta_{6} w_{i k}\right], \\
c_{1}(t) \geq 0 \\
c_{2}(k) \geq 0 \\
c_{3}(k) \geq 0 \\
c_{4}(k) \geq 0 \\
c_{5}(k) \geq 0
\end{gathered}
$$

where $t$ is the propagation distance of shock wave and $\tilde{E}$ is the charge quantity:

$$
\begin{gathered}
\bar{t}=\frac{t}{\sqrt[3]{\widetilde{E}}}, \\
P\left(d_{i}, w_{j}\right)=P\left(d_{i}\right) P\left(w_{j} \mid d_{i}\right) ; P\left(w_{j} \mid d_{i}\right) \\
=\sum_{k=1}^{K} P\left(w_{j} \mid z_{k}\right) P\left(z_{k} \mid d_{i}\right), \\
\frac{\partial \pi_{B}^{L H}}{\partial p_{2}}=\frac{1-p_{2}+p_{1}}{2}-\frac{p_{2}-c_{2}}{2}+\frac{k-p_{2}+p_{1}}{2 k} \\
-\frac{p_{2}-c_{2}}{2 k}-e t\left[-\frac{1}{2}-\frac{1}{2 k}\right]=0 .
\end{gathered}
$$

The above formula is the proportional distance; $\mathrm{S}_{0}, S_{1}, S_{2}$, etc. are determined by the test results $[13,14]$.
TABLE 1: Basic test parameters of the shock wave storage test system.

\begin{tabular}{lcccccc}
\hline Item & Reliable & Accurate & Error & Stable & Multiple & Frequency \\
\hline 1 & 1.38 & 1.14 & 0.06 & 0.37 & 1.93 & 1.43 \\
2 & 1.66 & 3.97 & 3.85 & 3.84 & 3.34 & 1.13 \\
3 & 4.51 & 5.76 & 5.06 & 2.25 & 5.38 & 5.41 \\
4 & 1.03 & 1 & 1.04 & 2.64 & 1.18 & 4.09 \\
\hline
\end{tabular}

For the air explosion of naked TNT and spherical explosive, the maximum pressure has the following expression:

$$
\Delta A=\frac{0.0086}{\bar{t}}+\frac{0.0035}{\bar{t}^{2}}+\frac{0.75}{\bar{t}^{3}},
$$

$\min w_{k}(t)=\left[\omega_{1}\left(\frac{d_{k}}{V}\right)+\omega_{2}\left(\frac{d_{k}}{V}\right)+\omega_{3}\left(\frac{T_{k}}{N D_{K}}\right)+\omega_{1}\left(P_{K} T_{K}\right)\right]$.

2.3. Calculation Method of Action Time in Barotropic Zone. In the case of air explosion, the duration of positive pressure of shock wave is determined by energy $R_{0}$, initial pressure $p_{0}$, initial air density $\rho_{0}$, and ignition distance $t$. Therefore, it can be expressed as

$$
\begin{gathered}
\delta=G\left(R_{0}, P_{0}, \rho_{0}, t\right), \\
\wp_{\kappa}=\frac{2 k}{k+1}+\left[\frac{1}{2}+\frac{1}{2 k}\right]\left[\frac{c_{2}-c_{1}}{3}\right]^{2}+\frac{2\left(c_{2}-c_{1}\right)}{3}, \\
y=\alpha W y+\beta_{1} X-W \beta_{2} X+\varepsilon .
\end{gathered}
$$

It is also available.

$$
\begin{gathered}
\frac{\delta}{\sqrt[3]{\tilde{E}}}=G_{1}\left(\frac{t}{\sqrt[3]{\tilde{E}}}\right) \\
w_{i k}=\sum_{a}^{n} \tau_{1} X_{i k}+\sum_{b}^{n} \tau_{2} U\left(Y_{i k}\right)+B_{i k} .
\end{gathered}
$$

2.4. Fluent Processing Method. Fluent is a special software for numerical simulation and analysis of fluid movement in and out of the complex geometry area and analysis of experimental simulation data. Fluent supports a wide range of network types, enabling users to solve problems smoothly under the premise of ensuring the accuracy of experimental data [15]. Fluent also provides a variety of physical models and the best numerical solutions, so that users can obtain satisfactory computing speed, stability, and accuracy. The process can monitor the simulation process and calculation results in real time through multiple operations [16]. It has a variety of display and storage modes, such as cloud, contour, vector, and animation. It can store results in the format supported by the processing software. It includes three basic functions: preprocessing, solver, and postprocessing.

(1) Preprocessing includes gambit, tgrid, and filters, which are used to model and process simulation objects; grid can import uploaded geometric patterns 


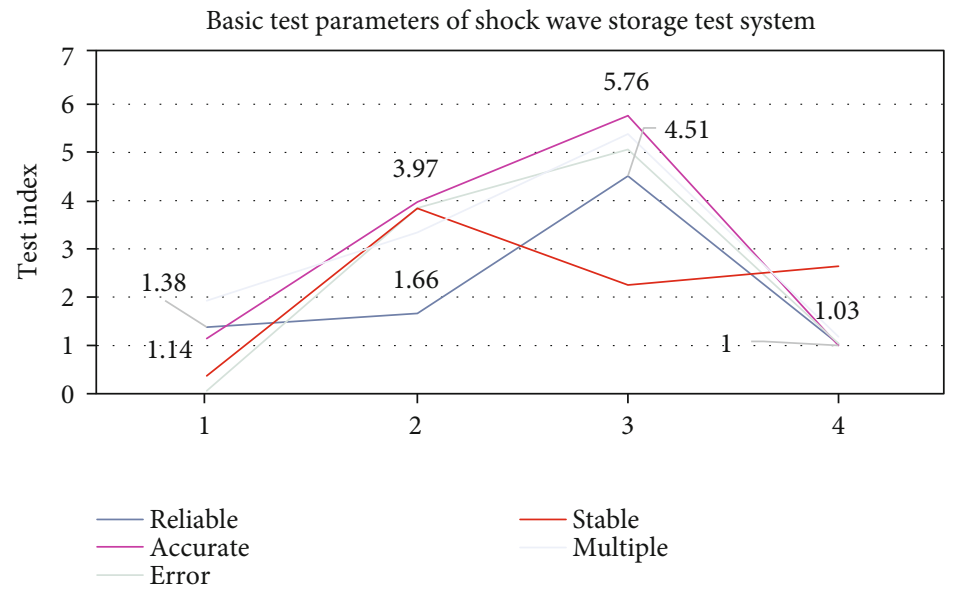

FIGURE 1: Basic test parameters of the shock wave storage test system.

on gambit software; filters are subsystems of another CAD or CAE and can read the network composed of them $[17,18]$

(2) The solver is the basic part of fluent. After reading the grid file correctly, the tasks of setting boundary conditions, material selection, numerical solution, mesh optimization, and simple postprocessing can be easily completed

(3) The postprocessor flow software provides cloud, vector, contour, and animation to display the calculation results. It can also enlarge, translate, and rotate the data results and export the current results to other postprocessing software (such as tecpart) for editing $[19,20]$

2.5. AD Conversion Method. AD inverter is an important part of the whole test system, which is related to the acquisition rate and accuracy of the signal. The principle of $\mathrm{AD}$ conversion is as follows: the first step of converting analog signal $F(t)$ into digital signal $F(n)$ is to sample $F(t)$ at fixed intervals $[21,22]$. The sampling value $f_{\mathrm{s}}$ (NTS) is obtained. The TS parameter is the sampling period, and the reciprocal is the sampling frequency $f_{s}$. According to Nyquist sampling theorem, if the original signal is to be completely recovered from the sampled signal, $f_{\mathrm{s}} \geq 2 f_{\mathrm{m}}$ must be satisfied [23]. The highest frequency of the signal is $f_{\mathrm{m}}$, and $f_{\mathrm{s}}$ (NTS) is quantized into $F_{q}\left(n T_{\mathrm{s}}\right)$ signal. Finally, digital signal $F(n)$ is received through a coding process to convert analog signal into digital signal $[24,25]$.

\section{Shock Wave Storage Test System Design Experiment}

3.1. Sensor Adaptation Circuit Design. In addition to the constant voltage and current circuit, the analog system also has a linear LDO power supply, which mainly generates $8 \mathrm{~V}$ constant voltage for multiple voltage stabilizing circuits and filter circuits and $1.65 \mathrm{~V}$ bias voltage for system use. In order to reduce power ripple, lp2985 is used as the main component
TABLE 2: Error test results of adaptation circuit.

\begin{tabular}{lcccccc}
\hline \multicolumn{3}{c}{ Working parameters } & \multicolumn{5}{c}{ Actual output } \\
Gain & $\begin{array}{c}\text { Frequency } \\
\left({\left.\mathrm{Sa} \cdot \mathrm{s}^{-1}\right)}^{\text {Input }}\right.\end{array}$ & $\begin{array}{c}\text { Soltage } \\
V_{\mathrm{pp}}(\mathrm{V})\end{array}$ & $\begin{array}{c}\text { Sampling } \\
\text { frequency } \\
\left(\mathrm{Sa} \cdot \mathrm{s}^{-1}\right)\end{array}$ & $\begin{array}{c}\text { Output } \\
\text { voltage } \\
V_{\mathrm{pp}}(\mathrm{V})\end{array}$ & Gain & $\begin{array}{c}\text { Error } \\
(\%)\end{array}$ \\
\hline 0.125 & $3 \mathrm{M}$ & $6 \mathrm{M}$ & $3 \mathrm{M}$ & 0.635 & 0.127 & 0.14 \\
0.25 & $3 \mathrm{M}$ & $6 \mathrm{M}$ & $3 \mathrm{M}$ & 1.037 & 0.263 & 0.53 \\
0.5 & $2 \mathrm{M}$ & $4 \mathrm{M}$ & $2 \mathrm{M}$ & 1.274 & 0.561 & 1.22 \\
1 & $2 \mathrm{M}$ & $2 \mathrm{M}$ & $2 \mathrm{M}$ & 0.926 & 1.027 & 0.35 \\
\hline
\end{tabular}

of linear power supply. The chip has a large dynamic range input and can achieve $8.5 \mathrm{~V}$ to $10 \mathrm{~V}$ power conversion, corresponding to peripheral resistance and capacitance.

3.1.1. Adjustable Amplifier Circuit. The output of ICP sensor is $-8 \sim+10 \mathrm{~V}$ AC signal, which is based on $10 \sim 15 \mathrm{~V}$ DC voltage. It is usually input to operational amplifier circuit after AC connection through a capacitor. The voltage sequence is designed by opa2340 and manufactured by TI company. It has the characteristics of single power supply, rail input and output, high swing speed, high input, and low output, and the former circuit is in high-strength state, and the last circuit is on low-intensity conditions, so that the front and back stage circuits can be isolated and stored.

3.1.2. Power Amplifier Circuit. In this paper, $10.5 \mathrm{~V}$ lithium battery is used as the power supply of the system. In order to meet the driving requirements of ICP piezoelectric sensor, a DC-DC boost power supply circuit and DC power supply circuit are designed. Using the tps670 power level chip and the corresponding resistance and capacitance design, the power supply can be converted from $10.5 \mathrm{~V}$ to $36 \mathrm{~V}$, and the constant current can be realized by a constant current diode.

3.2. Physical Model of Shock Wave Overpressure Tester and Related External Flow Settings. The general steps to determine the calculation model are as follows: 


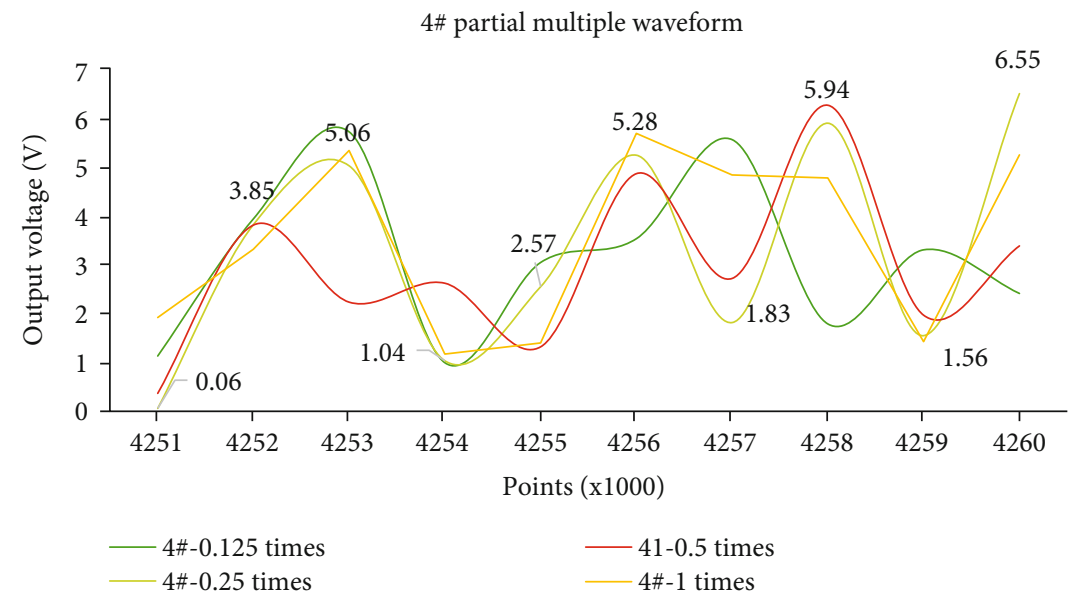

FIgURE 2: 4\# partial multiple waveform.

(1) Create 3D geometry (cube, cone, cylinder, etc.), and divide and cut geometry according to actual structure

(2) Create mesh: divide the border of the geometric structure and the lines of the external area of the structure into isometric or nonisometric network, and then, create the line network as the face network

(3) Determine input and output constraints and solid walls, and store network files

According to the above steps, the external flow field of the disc and pen piezometers is formed as follows: the main body of the impact pipe is constructed, and the simplified cylinder model is determined according to the actual size of the lowpressure chamber $(6500 \mathrm{~mm})$. The length and radius are $60 \mathrm{~mm}$; the structural model of the sensor is specified, and the cylindrical and conical models are moved to the actual installation position, and the impact pipe and sensor group are moved to the actual installation position. External flow entity modeling and related settings of shock wave overpressure storage and testing device are used.

3.3. Setting External Flow Problems. The external flow around the disc and pen gauges has been specially activated. After making the model with gambit software, the grid file is inserted into fluent solver for analysis. Since this is a threedimensional problem, the three-dimensional and onedimensional accuracy is selected to solve the problem. The decomposition steps are as follows:

(1) Mesh relevancy: check the mesh to ensure that the minimum mesh volume is not negative, otherwise the mesh will be reused; set the unit size to millimeter; smooth and swap the mesh

(2) Selection model calculation: coupling solver is usually used in high-speed aerodynamics; S-A turbulence model is selected for liquid air problems with wall conditions

(3) Material properties: when Mach number is greater than 0.5 , it can be considered as compressed fluid.
TABLE 3: Flexibility and adjustable magnification of the designed adaptive.

\begin{tabular}{lcccc}
\hline Points & $4 \#-0.125$ times & 4\#-0.25 times & 4\#-0.5 times & $4 \#-1$ times \\
\hline 4251 & 1.14 & 0.06 & 0.37 & 1.93 \\
4252 & 3.97 & 3.85 & 3.84 & 3.34 \\
4253 & 5.76 & 5.06 & 2.25 & 5.38 \\
4254 & 1 & 1.04 & 2.64 & 1.18 \\
\hline
\end{tabular}

Considering its compressibility and thermophysical properties, the calculated viscosity can be adjusted by Saran's law and open energy equation

(4) Because it is related to the total pressure, static pressure, and other pressures, setting the working pressure to 0 is convenient for setting the limit and posttreatment conditions, and the influence of gravity is not considered

(5) Determine the boundary conditions: in this paper, the wall and shape structures of the impact pipe are the wall conditions. The pressure value of shock tube, the corresponding Mach number and pressure value, and the setting of flow element in the $X Y$ direction must be adjusted according to the actual situation

(6) Control parameters: when the convergence rate is slow and stable, increase the Coulomb number and increase the relaxation factor to improve the convergence rate

(7) Initialize the flow field and specify the number of iterations to start the iteration operation

3.4. Big Data Evaluation Test and Database Parameter Data Collection. With the introduction of big data theory and design, cloud computing, and related MapReduce and HBase kernel technology, Hadoop has been determined to be born from big data. Then, the Hadoop platform is used to verify the ability of processing and evaluating case test data based on the big data technology proposed in this paper. 


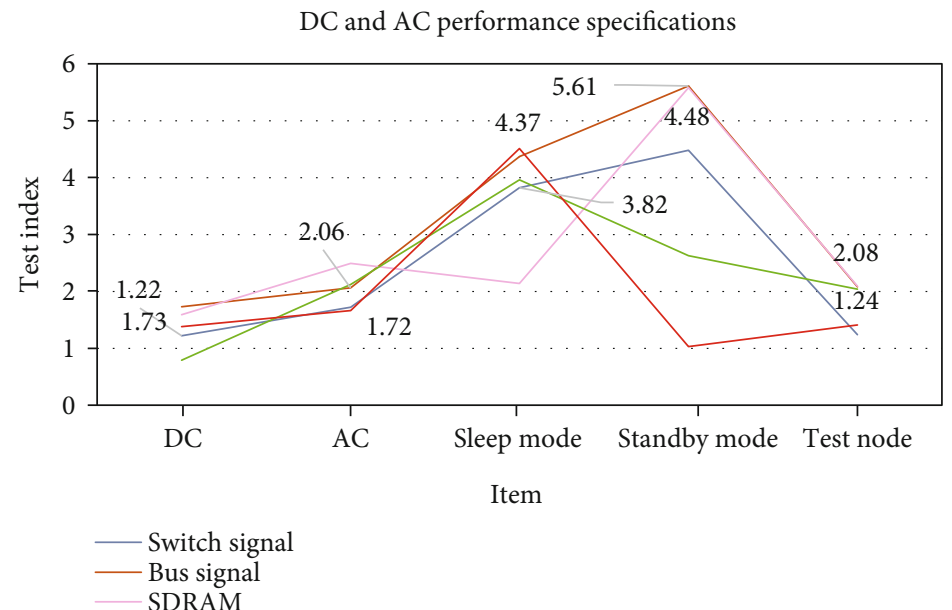

Figure 3: DC and AC performance specifications.

Table 4: Converted digital signal is mounted on the data line.

\begin{tabular}{lccccc}
\hline Item & $\begin{array}{c}\text { Switch } \\
\text { signal }\end{array}$ & $\begin{array}{c}\text { Bus } \\
\text { signal }\end{array}$ & SDRAM & FPGA & $\begin{array}{c}\text { Power } \\
\text { consumption }\end{array}$ \\
\hline DC & 1.22 & 1.73 & 1.59 & 0.79 & 1.38 \\
AC & 1.72 & 2.06 & 2.49 & 2.12 & 1.66 \\
$\begin{array}{l}\text { Sleep } \\
\text { mode }\end{array}$ & 3.82 & 4.37 & 2.14 & 3.96 & 4.51 \\
$\begin{array}{l}\text { Standby } \\
\text { mode }\end{array}$ & 4.48 & 5.61 & 5.58 & 2.63 & 1.03 \\
Test node & 1.24 & 2.08 & 2.09 & 2.04 & 1.41 \\
\hline
\end{tabular}

\section{Design and Analysis of Shock Wave Storage Test System}

4.1. Performance Test and Analysis of Sensor Adapter Circuit. In order to test the reliability and accuracy of the adaptive circuit, the signal generator is used to debug the designed circuit system (in order to better test the system error, the original multiple is reduced to 0.125 times of the original setting). The basic test data is shown in Table 1 and Figure 1.

LabVIEW human-computer interaction interface is used to show the corresponding waveform change diagram of 4 $\times 10$ (20 sets of devices) at 0.125 times, 0.25 times, 0.5 times, and 1 time. The amplitude of output voltage under different multiples can be read out at the software interface $\Delta x y$. Table 2 shows the test results of the $4 \times 10$ device, which shows that the error of the adaptive circuit is less than $2 \%$. Compared with the traditional circuit, the system error is greatly reduced and the test accuracy is improved.

As shown in Figure 2, this paper designs a special power boost circuit, an adjustable amplification circuit, a bias circuit, and a filter circuit. In the natural environment, using the human-computer interface of the special software LabVIEW, the circuit parameters of the randomly selected $4 \times$ 10 device are analyzed, and the waveform diagram of the 4 $\times 10$ device at 0.125 times, 0.25 times, 0.5 times, and 1 time is given. As shown in Table 3, the results show that the error is less than $2 \%$, and the flexibility and adjustable magnification of the designed adaptive circuit are verified.

\subsection{Realization of Free-Field Shock Wave}

4.2.1. AD Circuit. This research mainly uses an $\mathrm{AD}$ converter to realize the key technology of free-field shock wave, its task is to realize the analog signal without distortion digitization, and conversion accuracy is the core of the design of $\mathrm{AD}$ circuit. However, many factors will affect its accuracy, such as bits, quantization error, reference voltage, component deviation and temperature, and sampling frequency. In order to ensure the test accuracy of the system, it is necessary to select a suitable analog-to-digital converter.

As shown in Figure 3, Ad7482 uses advanced technology to achieve extremely low integral nonlinear error and offset and gain error and has excellent DC and AC performance specifications. In addition, it also provides two power saving modes: sleep mode and standby mode. Even in the working mode, the power consumption is only $90 \mathrm{mw}$, which is suitable for the design of miniaturized test node, and its typical circuit design is adopted.

As shown in Table 4, when convst signal is in a low level, start sampling and analog-to-digital conversion, and then, the bus signal becomes high and keeps at a high level during conversion. Once the conversion is finished, the bus signal is reset to a low level, and the converted digital signal is mounted on the data line, which is convenient for FPGA to read or store in SDRAM.

4.2.2. Memory Circuit Test. As shown in Figure 4, the storage circuit caches the converted digital signal to the memory, which is convenient for data recovery. It is the key method to obtain the explosion environment signal. The core of the circuit is data memory, which transfers data from the AD converter to memory and from memory to the output interface. The power supply voltage of the storage circuit is $3.3 \mathrm{~V}$. The memory chip adopts synchronous dynamic memory $\mathrm{mt} 48 \mathrm{lc} 8 \mathrm{~m}$, and the working interface mainly includes data bit, address bit, write/read control, and write/read clock. Dynamic memory also needs strict timing to realize the 


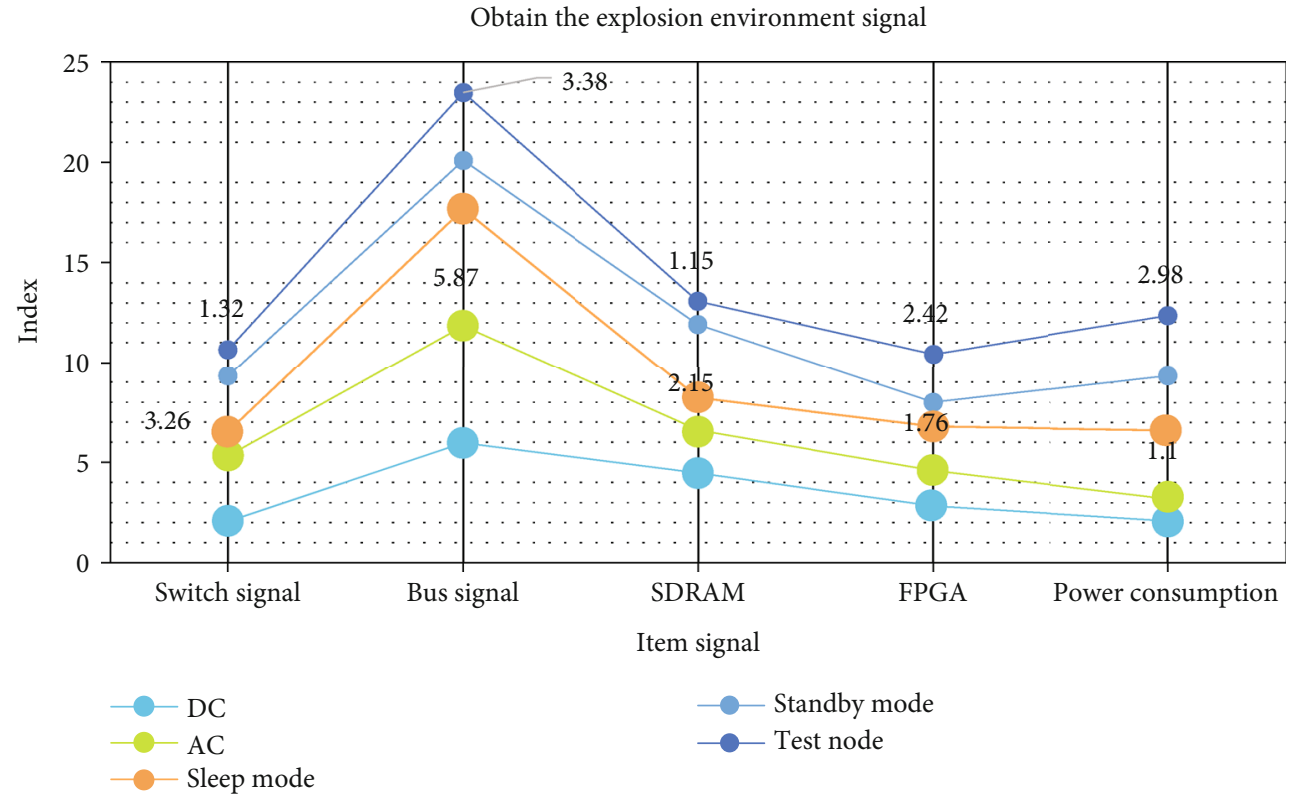

FIGURE 4: Obtain the explosion environment signal.

TABLE 5: Dynamic memory also needs strict timing to realize the functions.

\begin{tabular}{lccccc}
\hline Item & DC & AC & $\begin{array}{c}\text { Sleep } \\
\text { mode }\end{array}$ & $\begin{array}{c}\text { Standby } \\
\text { mode }\end{array}$ & $\begin{array}{c}\text { Test } \\
\text { node }\end{array}$ \\
\hline Switch signal & 2.05 & 3.26 & 1.22 & 2.8 & 1.32 \\
Bus signal & 5.97 & 5.87 & 5.83 & 2.43 & 3.38 \\
SDRAM & 4.46 & 2.15 & 1.62 & 3.69 & 1.15 \\
FPGA & 2.82 & 1.76 & 2.2 & 1.22 & 2.42 \\
Power & 2.08 & 1.1 & 3.42 & 2.75 & 2.98 \\
consumption & & & & &
\end{tabular}

functions of writing, reading, and refreshing. As shown in Table 5, the system is implemented by FPGA, which is responsible for connecting the data bus of memory.

4.3. Pressure Time Curve Analysis. In this paper, the pressure time curve measured by the test device is directly read, and then, the value of shock wave overpressure is obtained. Taking two sets of devices as examples, their pressure time curves are shown in Figure 5.

As shown in Figure 5, it can be seen that before the arrival of shock wave overpressure, a lot of jitters are superimposed on the baseline of the test system. This is because the velocity of some fragments exceeds the velocity of shock wave in the pressure measuring area installed by the test device during explosion, and these fragments pass through the air at supersonic speed to form a projectile track shock wave; in response to the sensor, there are many small pressure disturbances overlapping on the time-varying curve of shock wave pressure.

4.4. Comparison and Analysis of External Flow Field Simulation Results of Two Kinds of Sensors. The disc type and pen type shape structures are simulated with multiple pressure values under different concave-convex heights and different deviation angles. The results are shown in Figure 3. The abscissa in the figure is the Mach number corresponding to the inlet pressure (calculated by using fluent modeling), and the ordinate is the measured pressure value. Different lines are used to represent the different heights and angles of the two structures, which is very good. It reflects the influence of different installation methods of the two structures on the test results. When the step pressure wave generated by the shock tube reaches the sharp corner of the windward surface of the disk, the shock wave below the disk plane flows through the disk plane according to the original propagation direction and passes through the sensor sensitive surface. The pressure change of the sensitive surface is very small. The shock wave above the disk plane reflects when it meets the rigid wall and forms an eddy current at the intersection of the vertical plane and inclined plane, the maximum pressure and density are $619394 \mathrm{pa}$, and a weak bow wave is formed at the sharp corner of the disk under the influence of the eddy current. With the propagation of shock wave, the eddy current range extends outward, and the shock wave propagates upward rapidly after passing through the disk plane of leeward surface. Under the influence of the shape structure, the vortex is formed. The pressure value decreases rapidly, and the minimum value reaches $25159 \mathrm{pa}$. In the simulation process, there is even negative pressure at the vortex, and there is flow separation phenomenon. From the pressure curve of the monitoring surface, it can be seen that the pressure change of the sensitive surface of the sensor is very small, close to the inlet pressure, indicating that the shock wave inflow is relatively stable. It can be seen from the same figure that the velocity of the incident flow and the reflected wave decreases sharply at the vertical plane of the configuration structure, and the minimum velocity is 0.55 mach. The maximum pressure changes in the flow field are at the windward side and leeward side of 


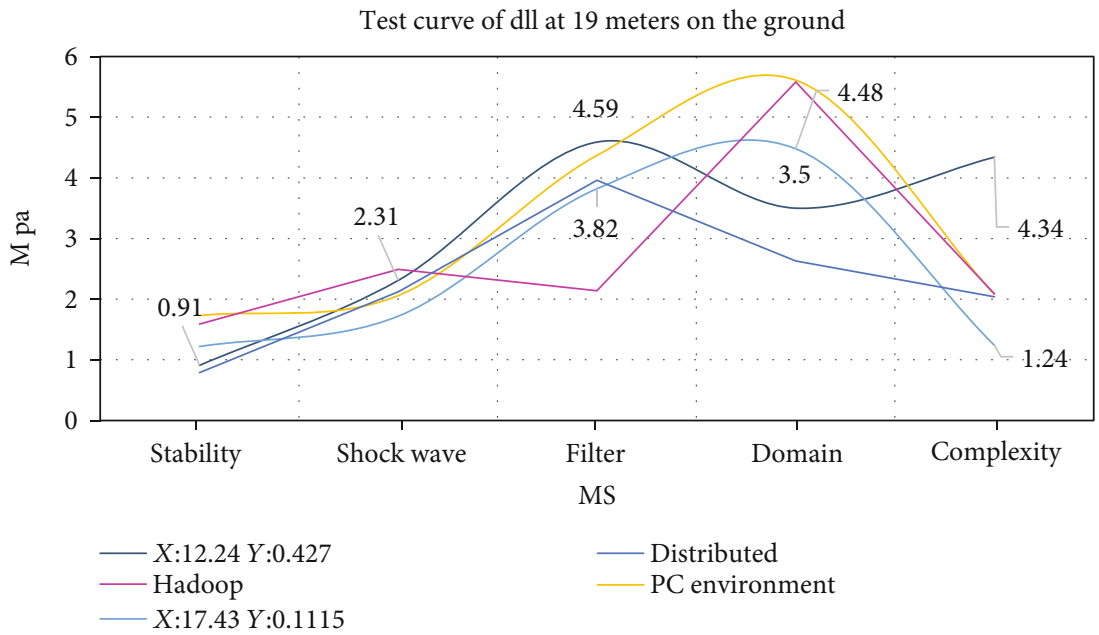

Figure 5: Test curve of $\mathrm{d} 11$ at 19 meters on the ground.

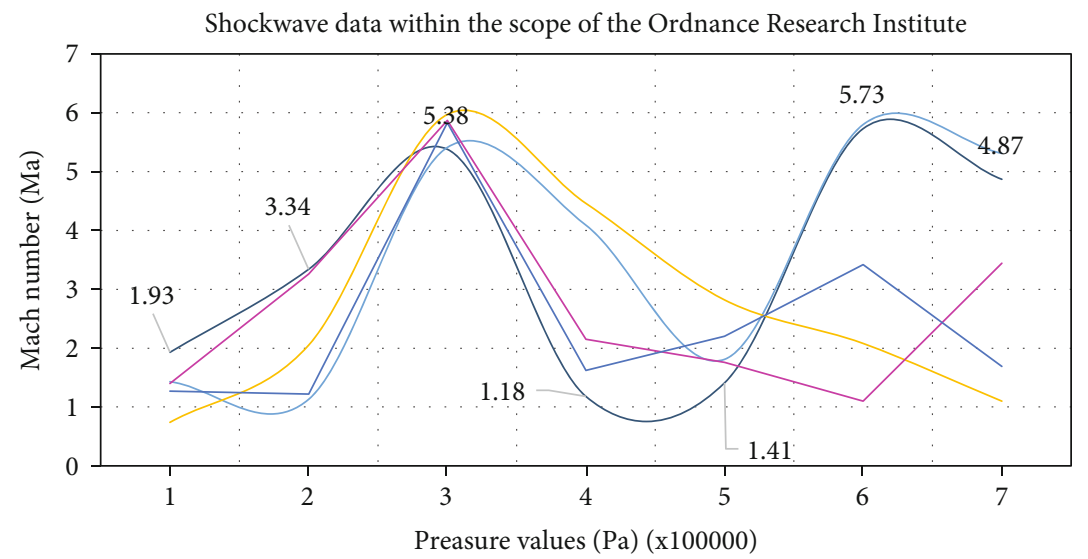

FIgURE 6: Histogram of comparison between the disc and pen bump.

TABLE 6: Two shape structures on the test results increase.

\begin{tabular}{lccccc}
\hline Num & $\begin{array}{c}\text { Disc } \\
\text { bump }\end{array}$ & $\begin{array}{c}\text { Pen } \\
\text { bump }\end{array}$ & $\begin{array}{c}\text { PC } \\
\text { environment }\end{array}$ & Hadoop & Distributed \\
\hline 1 & 1.93 & 1.43 & 0.74 & 1.4 & 1.27 \\
2 & 3.34 & 1.13 & 2.05 & 3.26 & 1.22 \\
3 & 5.38 & 5.41 & 5.97 & 5.87 & 5.83 \\
4 & 1.18 & 4.09 & 4.46 & 2.15 & 1.62 \\
5 & 1.41 & 1.81 & 2.82 & 1.76 & 2.2 \\
6 & 5.73 & 5.8 & 2.08 & 1.1 & 3.42 \\
7 & 4.87 & 5.31 & 1.1 & 3.44 & 1.69 \\
\hline
\end{tabular}

the disk slope and the vertical pipe wall, respectively, and the pressure change is very small at the plane disk.

It can be seen from Figure 6 that when the two structures have the same protrusion height or depression depth, the influence of the two shape structures on the test results increases with the increase of the inlet pressure. For the same inlet pressure, the larger the bulge or depression value, the greater the impact on the results.

As shown in Table 6, the influence of the disk is greater than that of the pen type, and the change rate of the relative difference increases with the increase of the concave-convex value. At the same angle deviation, the influence of the two shape structures on the test results increases with the increase of the inlet pressure. At the same inlet pressure, the larger the deviation angle, the greater the impact on the results. The influence of the disk is greater than that of the pen type, and with the increase of the angle value, the change rate of the relative difference also increases.

4.5. System Computing Performance Test. As shown in Figure 7 , the data evaluation model algorithm designed in this paper has high accuracy, but it will generate more intermediate results and temporary matrix in the calculation and execution, and the time complexity reaches $O\left(n^{2} \log (n)\right)$, which is too much for an ordinary single machine environment processing platform, especially when the amount of data is large. By referring to a large number of data, the big data processing technology is introduced, as shown in Table 7.

The massive shock wave test data and calculation processing tasks are distributed on the cluster computer to solve the dilemma of a single machine mode, so as to improve the calculation efficiency and accuracy. Based on the measured 


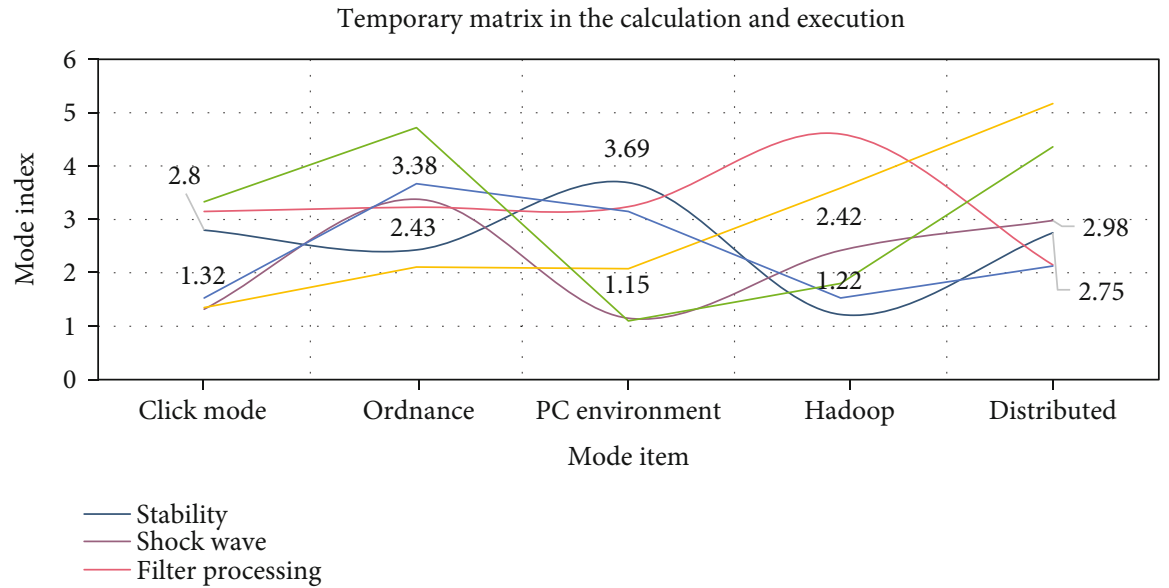

Figure 7: Temporary matrix in the calculation and execution.

TABLE 7: Big data processing technology is introduced.

\begin{tabular}{|c|c|c|c|c|c|}
\hline Item & Shock wave & Filter processing & Domain analysis & Complexity & Temporary matrix \\
\hline Click mode & 1.32 & 3.15 & 1.35 & 1.53 & 3.33 \\
\hline Ordnance & 3.38 & 3.23 & 2.11 & 3.67 & 4.72 \\
\hline PC environment & 1.15 & 3.24 & 2.08 & 3.15 & 1.1 \\
\hline Hadoop & 2.42 & 4.6 & 3.59 & 1.53 & 1.8 \\
\hline Distributed & 2.98 & 2.15 & 5.17 & 2.13 & 4.36 \\
\hline
\end{tabular}

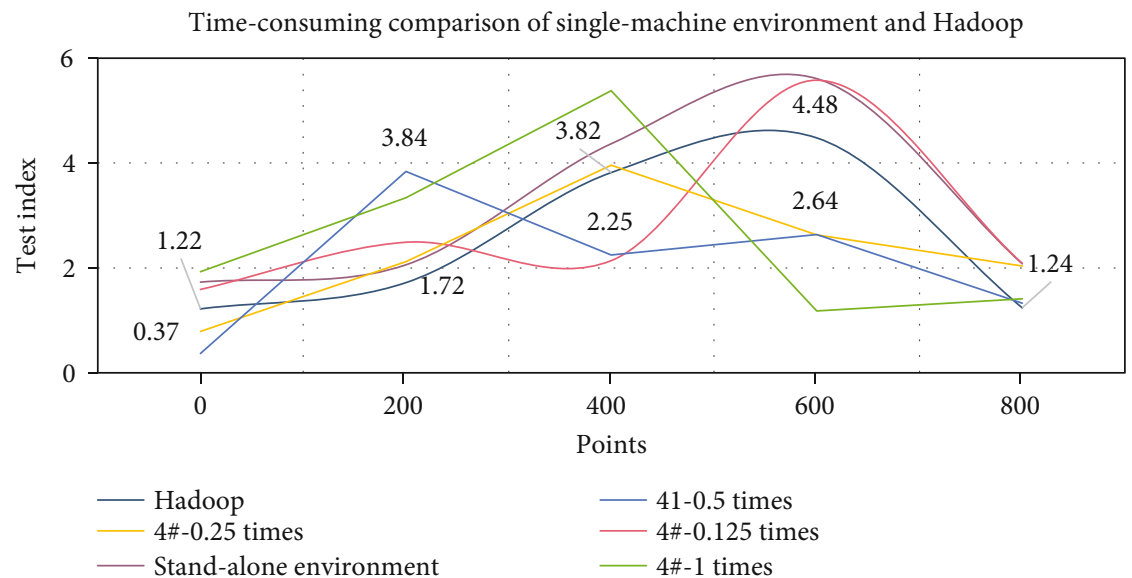

FIGURE 8: Time-consuming comparison of single-machine environment and Hadoop filtering.

TABLE 8: Hadoop needs more initialization preparation and intermediate.

\begin{tabular}{lccccc}
\hline Item & Click mode & Ordnance & PC environment & Hadoop & Distributed \\
\hline Stability & 0.91 & 1.22 & 1.73 & 1.59 & 2.49 \\
Shock wave & 2.31 & 1.72 & 2.06 & 2.14 & 2.12 \\
Filter & 4.59 & 3.82 & 4.37 & 5.61 & 5.58 \\
Domain & 3.5 & 4.48 & 2.08 & 2.09 & 2.63 \\
Complexity & 4.34 & 1.24 & & & 2.04 \\
\hline
\end{tabular}


shock wave data in the range of Ordnance Research Institute, this part compares the time required for frequency domain decomposition and filtering processing under the traditional single PC environment and Hadoop distributed platform of the system.

As shown in Figure 8, through the above analysis, when the amount of data is small, Hadoop consumes more time because the MapReduce framework cannot open multiple nodes to process the calculation process, and the whole calculation is implemented on one node (equivalent to standalone mode).

As shown in Table 8, Hadoop needs more initialization preparation and intermediate result transmission time, so it consumes more time. When the amount of data reaches a certain scale, Hadoop will start the cluster node distributed processing the same computing process, and its advantages will naturally be highlighted.

\section{Conclusions}

In the destruction process of weapon warhead, explosion shock wave is one of the essential characteristic physical quantities, which can be measured accurately, which can provide some reference data for the research of weapon destructive power. However, in the actual shock wave test, the amount of test data increases rapidly, and the data structure and types are more and more. The traditional test procedures and data processing technology cannot meet the requirements of rapid testing and cannot meet the most valuable test evaluation results in sea level data. In the current research process, the accuracy and intelligence of the test need to be improved. In this paper, the basic technology and realization of free-field shock wave are completed. It includes AD circuit, storage and recording module, activation circuit, interface circuit, power management module, computer management system, and data processing system. We introduce the implementation of synchronization module and verify the errors of single and multiple synchronization units. Special circuit installation and protection structure are designed for the developed system structure, so as to avoid direct damage to test equipment due to bullet.

In this paper, a test method is studied. The rationality of overpressure value is tested by measuring the velocity of shock wave and directly measuring the overpressure time curve of shock wave. In the process of research and development of the test system, the wireless device complements the functions of flash data storage, gain amplification, frequency increase, retrigger, activation, and internal power generation adjustment, so as to effectively improve the system reliability, operation simplicity, and practicability. The dynamic characteristics of the test system are analyzed, and the performance index and operation index of the test system are verified by the actual explosion test.

In this paper, fluent fluid dynamics simulation software and impact tube are used to study the influence of the convexity and deflection angle of the sensor sensitive surface on the pen and disc test results. It focuses on the simulation of different convex angle, different convex height, depression depth, and installation error under different insertion angles.
The conclusion is that the two shape structures meet the safety requirements. Under the same conditions, the error measured by the pen structure is less than that of the disk structure, while the error caused by concave and convex installation is far greater than that caused by claim of disc structure. What is the reason for this? The simulation results of the broken line method are more intuitive. The results show that the influence of the two schematic structures on the test results increases with the installation angle or the concave-convex degree and increases with the increase of the concave-convex degree. The influence of the angle on the test results is that the pen structure has less influence on the test results than the disk structure, so the pen structure is used for dynamic calibration of the two structures under different installation conditions next. The experimental data and simulation results are compared and analyzed to verify the accuracy of the simulation results.

\section{Data Availability}

No data were used to support this study.

\section{Conflicts of Interest}

The authors declare that they have no conflicts of interest.

\section{Acknowledgments}

This work was supported by the project future school (infant education) of National Center for Schooling Development Programme of China (grant no. CRIKT201912); Natural Science Foundation Project of Chongqing, Chongqing Science and Technology Commission (cstc2017jcyjAX0092); the Scientific and Technological Research Program of Chongqing Municipal Education Commission (KJQN201801601), Scientific Research Project of Chongqing University of Education (ky201926c) and the Science and Technology Research Program of Chongqing Municipal Education Commission (Grant No KJQN201801606).

\section{References}

[1] M. Robert, B. Segui, C. Vergnes, P. Taourel, and J. Guiter, "Piezoelectric extracorporeal shockwave lithotripsy of distal ureteric calculi: assessment of shockwave focusing with unenhanced spiral computed tomography," BJU International, vol. 87, no. 4, pp. 316-321, 2015.

[2] J. S. Couldrick, S. L. Gai, J. F. Milthorpe, and K. Shankar, "Normal shock wave/turbulent boundary-layer interaction control using 'smart' piezoelectric actuators," in Aeronautical Journal, vol. 109 , no. 1101 pp. $577-583$, 10.1017/s0001924000000919, 2016.

[3] J. D. Tobin and M. J. Hargather, "Quantitative schlieren measurement of explosively-driven shock wave density, temperature, and pressure profiles," Propellants Explosives Pyrotechnics, vol. 41, no. 6, pp. 1050-1059, 2016.

[4] M. Bai, Y. Zhao, B. Jiao, X. Zhai, and Y. Geng, "A novel easydriving and easy-signal-processing electrostatic field sensor based on a piezoresistance and polyethylene terephthalate 
lever," Journal of Micromechanics \& Microengineering, vol. 27, no. 3, article 035002, 2016.

[5] C. Z. Peng and C. K. How, "Giant retroperitoneal abscess following extracorporeal shock wave lithotripsy," Internal Medicine, vol. 54, no. 24, pp. 3251-3252, 2015.

[6] S. M. Han and C. S. Huh, "Analysis of a piezoelectric generator under an elastic wave," IEEE Transactions on Plasma Science, vol. 45, no. 11, pp. 3001-3006, 2017.

[7] F. Castro, T. Pentiado, J. Blanco, R. Xavier, M. Sanches, and A. de Carvalho, "Crosstalk error analysis in IIDFC readout circuit for use in piezoresistive composite," IEEE Sensors Journal, vol. 18, no. 1, pp. 382-389, 2018.

[8] K. Winston, June Heatwave Could Trigger Aliso Storage Withdrawals: California Official, Platts Energy Trader, 2019.

[9] K. M. Thijs, J. Zwerver, F. J. G. Backx et al., "Effectiveness of shockwave treatment combined with eccentric training for patellar tendinopathy: a double-blinded randomized study," Clinical Journal of Sport Medicine, vol. 27, no. 2, pp. 89-96, 2017.

[10] S. Raghunathan and D. G. Mabey, "Passive shock-wave/boundary-layer control on a wall-mounted model," AIAA Journal, vol. 25, no. 2, pp. 275-278, 1987.

[11] L. Li, D. Qian, X. Zou, and X. Wang, "Underwater electrical wire explosion: shock wave from melting being overtaken by shock wave from vaporization," Physics of Plasmas, vol. 25, no. 5 , article $053502,2018$.

[12] C. Sudhiesh Kumar and K. P. J. Reddy, "Experiments in handoperated, hypersonic shock tunnel facility," Shock Waves, vol. 26, no. 6, pp. 845-849, 2016.

[13] J. S. Couldrick, S. L. Gai, J. F. Milthorpe, and K. Shankar, "Investigation of unswept normal shock wave/turbulentboundary-layer interaction control," Journal of Aircraft, vol. 46, no. 5, pp. 1634-1641, 2015.

[14] G. Thomas, J. Y. Chapelon, and C. Lafon, "Finite element optimization of circular element used in lithotripsy and histotripsy piezoelectric transducers," Journal of the Acoustical Society of America, vol. 141, no. 5, pp. 3719-3719, 2017.

[15] J. L. Edwards, W. S. Hinman, and C. T. Johansen, "Extracting mole fraction measurements from the visualization of a shock reflection," Journal of Visualization, vol. 22, no. 1, pp. 35-49, 2019.

[16] M. V. Antipov, I. V. Yurtov, A. Utenkov et al., "Piezoelectric method for measuring the parameters of shock-induced ejecta," Combustion Explosion and Shock Waves, vol. 54, no. 5, pp. 599-605, 2018.

[17] S. Cooppan and B. Skews, "Three-dimensional shock wave diffraction off a discontinuous edge," Shock Waves, vol. 27, no. 2, pp. 131-142, 2017.

[18] G. Nath and S. Singh, "Corrigendum to: "Flow behind magnetogasdynamic exponential shock wave in self- gravitating gas" [Int. J. Non-Linear Mech. 88 (2017) 102-108]," International Journal of Non-Linear Mechanics, vol. 89, p. 132, 2017.

[19] C. Chelem Mayigue and R. Groll, "Computational magnetohydrodynamic modeling of hypersonic flows with resolved shock wave diffusion," PAMM, vol. 16, no. 1, pp. 621-622, 2016.

[20] W. Johanns, C. Jakobeit, L. Greiner, and J. Janssen, "Ultrasound-guided extracorporeal shock wave lithotripsy of pancreatic ductal stones: six years' experience," Canadian Journal of Gastroenterology, vol. 10, no. 7, pp. 471-475, 1996.
[21] A. M. Blokhin, D. L. Tkachev, and A. V. Yegitov, "Local solvability of the problem of the van Der Waals gas flow around an infinite plane wedge in the case of a weak shock wave," Siberian Mathematical Journal, vol. 59, no. 6, pp. 960-982, 2018.

[22] A. S. Savinykh, G. V. Garkushin, G. I. Kanel', and S. V. Razorenov, "Evaluation of viscosity of $\mathrm{Bi}-\mathrm{Pb}$ melt $(56.5 \%-43.5 \%)$ by the width of a weak shock wave," High Temperature, vol. 6, no. 5, pp. 685-688, 2018.

[23] M. H. Radfar, N. Simforoosh, M. Sotoudeh et al., "What is the impact of extracorporeal shock wave lithotripsy on semen parameters? A systematic review and meta-analysis," Urologia Journal, vol. 84, no. 1, pp. 28-34, 2017.

[24] R. Assaly-Kaddoum, F. Giuliano, S. Compagnie, J. Bernabé, and D. Behr-Roussel, "Additive pro-erectile effect of low intensity-shockwave therapy (Li-ESWT) delivered by Aries combined with sildenafil in spontaneously hypertensive rats (SHR)," European Urology Supplements, vol. 16, no. 3, pp. e1945-e1946, 2017.

[25] T. Mimura, Y. Igarashi, K. Ito et al., "Efficacy of combined endoscopic treatments and extracorporeal shock wave lithotripsy for pancreatic lithiasis," Suizo, vol. 30, no. 2, pp. 154163, 2015. 